\title{
Conditioned suppression of operant responding in response to a stimulus paired with pentobarbital injections
}

\author{
PERRY M. DUNCAN \\ Old Dominion University, Norfolk, Virginia
}

\begin{abstract}
Conditioned suppression of ongoing behavior in response to a stimulus paired with barbiturate treatment was investigated in rats leverpressing for food pellets by presenting an audiovisual stimulus (conditioned stimulus [CS]) $3 \mathrm{~min}$ before injection of $15 \mathrm{mg} / \mathrm{kg}$ sodium pentobarbital (experimental group, $n=9$ ) or water (control group, $n=8$ ). The experimental rats completely stopped responding during the 20-min postinjection period. During 12 conditioning days, the experimental group developed conditioned suppression to the preinjection CS as indicated by decreases in response rate to $35 \%$ of baseline, significantly different from the $70 \%$ control group mean. The preinjection conditioned response lowered response rate, as did the actual drug treatment. Visual observation revealed that the CS elicited locomotor activity that interfered with leverpressing, indicating that although the conditioned response rate change was "isodirectional" to the drug effect (unconditioned response), an opposite-direction conditioned locomotor conditioned response was elicited by preinjection stimuli. The ability of the injection procedure to elicit conditioned responses was determined after 12 conditioning days in an extinction-test session in which water only was injected in both groups. Neither significant conditioned suppression nor conditioned motor activity was elicited by the injection procedure.
\end{abstract}

The investigation of conditioned responses to stimuli that predict the onset of psychoactive drug effects is an important topic in behavioral pharmacology. An interesting theoretical question involves the nature of the conditioned response (CR) in comparison with the unconditioned response (UR) produced by the actual drug administration. Drug-related CRs are sometimes isodirectional in relation to the UR ("drug-like"), and in other situations the CR is opposite in direction to that of the UR, possibly compensating for the drug effect. Theoretical accounts for these differences have been advanced (e.g., Eikelboom \& Stewart, 1982), but as Siegel (1989) has pointed out, the factors determining CR directionality are still not completely understood. Reviews of the experimental literature (cited below) suggest that isodirectional CRs are most often seen with central nervous system (CNS) stimulants (such as cocaine or amphetamine), whereas compensatory (opposite-direction) CRs are more often seen when CNS depressants or opiates are administered. There are, however, definite exceptions to this "drug type" classification of CR directionality. The complexity of the directionality question is further illustrated by reports of one CR (decreased motor activity), seen in animals anticipating insulin injection, being "drug-like" or isodirectional, simultaneously with another CR (blood glucose level changes) in the same ani-

Correspondence should be addressed to P. M. Duncan, Department of Psychology, Old Dominion University, Norfolk, VA 23529-0267 (e-mail: pmd200f@viper.mgb.odu.edu).

-Accepted by previous editor, Paul E Gold mals being of a compensatory nature (hyperglycemic; Duncan \& Koontz, 1994; Siegel, 1975).

Much of the previous research in this area has dealt with behavioral or physiological responses such as analgesia, body temperature changes, blood-glucose levels, motor activity, and drug-produced convulsions or lethality. The well-researched topics of conditioned taste aversion and conditioned place preference also obviously involve conditioned drug effects. For reviews of the experimental literature and discussion of theoretical concerns, see Siegel $(1983,1989)$ and Stewart and Eikelboom (1987).

Psychoactive drugs have complex (but highly reliable) effects on operant behavior, and response-rate-altering effects of drugs have been a major research area of behavioral pharmacology for many years. Response rate, as measured in an operant paradigm, is a specific, ongoing, easily measured behavior that can be very sensitive to drug effects, including drug-related CRs. The use of operant response rate to indicate conditioned emotional responses was introduced by Estes and Skinner (1941), who used footshock as the unconditioned stimulus (US) and a stimulus preceding the US as the conditioned stimulus (CS). This conditioned suppression paradigm has been adapted for the investigation of conditioned drug effects by using drug injection as the US (citations of previous work are listed below).

Operant response rate is an indirect indicator of a drug's primary effect on behavior, and the drug action that actually causes the response rate change may not be easily identifiable. For example, a decrease in responding could result from CNS depression involving ataxia or sedation or from drug-induced emotional or motivational states. Therefore, the value of response rate change as an indicator of the "di- 
rection" of the primary conditioned drug response may be limited if no other behavior or physiological responses are concurrently measured. However, the operant paradigm has the advantages of sensitivity and reliability, mentioned previously. In addition, there exists a large body of research literature derived from operant studies of drug effects that may be useful for evaluating the conditioned effects of drugs in this paradigm. Additionally, the relatively few previous studies using the operant paradigm to study conditioned drug responses have shown consistent results regarding the "direction" of these responses across a range of drugs, species, and schedules of reinforcement.

In earlier work, Cameron and Appel (1972) studied the effects of LSD-25 and chlorpromazine in rats; Whitney and Trost (1970) injected amphetamine IV in a monkey; and Goldberg and Schuster (1967) used a US consisting of injections of a narcotic antagonist in morphine-dependent monkeys. Two more recent studies of the effects of conditioned drug responses on operant response rate include the work of Duncan, Barry, Ellis, and Hinkle (1989), who administered amphetamine to rats, and Watanabe (1990), who studied the effects of barbiturate and amphetamine in pigeons.

In all of these experiments, the change in response rate produced by the $\mathrm{CR}$ was isodirectional to that produced by the actual drug injection. In most cases the CR and UR produced a suppression of response rate, but in the study of pigeons pecking for reinforcement on a fixed-interval (FI) schedule (Watanabe, 1990), the CR to amphetamine or barbiturate-paired stimuli resulted in increased response rates, as did the actual drug treatment.

No previous experiments have been reported in which rats performing an operant response are presented with a CS predicting the occurrence of barbiturate injection in such a manner that the development of the conditioningrelated response rate changes could be observed. In an experiment of this nature using amphetamine injection as the US (Duncan et al., 1989) an audiovisual stimulus preceded the drug injection, and conditioned suppression developed to both this stimulus and the stimulus of the injection procedure. The experiment presented here was of similar design, and was a further investigation of Pavlovian conditioning in the operant paradigm, using as a US injection of barbiturate at a dose sufficient to completely suppress leverpressing.

To better understand the nature of behavior elicited by the CS in the operant paradigm - that is, to deal with the "indirect" nature of response rate changes discussed above - systematic visual observations supplemented response rate as a second dependent variable. Although all previous experiments using the operant paradigm have demonstrated isodirectional (to the unconditioned drug effect) conditioned changes in response rate, the marked depressant effect of a moderate dose of barbiturate was expected to produce a compensatory CR. The direction of response rate changes accompanying this $\mathrm{CR}$ was of interest in the present experiment.

\section{METHOD}

\section{Subjects}

Seventeen male rats of the Long-Evans strain had food restricted until body weights reached $85 \%$ predeprivation level. Onset of illumination in the animal quarters was at $0700 \mathrm{~h}$, with offset at $1900 \mathrm{~h}$. All procedures were conducted between 1100 and 1500 .

\section{Apparatus}

Three Skinner boxes were located inside sound-attenuating enclosures and were equipped with a response lever, a houselight and cue lights, a loudspeaker, and a food pellet delivery mechanism. The dimensions of the operant chambers were $30.5 \times 25 \times 28 \mathrm{~cm}$ high. Programming modules controlled reinforcement delivery, and the total number of responses emitted each minute was recorded on a printing counter.

Injection solutions were sterile water and sodium pentobarbital (obtained from Sigma Chemical Co., St. Louis) dissolved in sterile water at a concentration of $15 \mathrm{mg} / \mathrm{ml}$.

\section{Procedure}

Operant training phase. The rats were trained to leverpress for 45-mg food pellets on a continuous reinforcement (CRF) schedule, and then shifted to a variable interval (VI) 60 -sec reinforcement schedule. A background masking noise (68-dB intensity white noise) was present during all sessions except during CS periods, as described below.

CS and injection adaptation. After response rates had stabilized, the rats were adapted to i.p. injections and to an audiovisual stimulus that would eventually predict drug or water injection. This adaptation was done by giving four i.p. water injections (volume $=$ $.5 \mathrm{ml}$ ) and four independent 3 -min stimulus presentations. One injection and one stimulus presentation were presented per day, and the stimulus did not predict the injection. These adaptation trials may have produced some latent inhibition that influenced the subsequent conditioning process (Lubow, 1973), but their inclusion was necessary to observe the development of the CRs in the absence of a marked orienting response to a novel stimulus.

At this point the subjects were assigned to the experimental group ( $n=9)$ or to the control group $(n=8)$, with baseline response rates and initial degree of suppression to water injection and to the audiovisual stimulus roughly matched between the two groups.

Conditioning phase. On each of 12 conditioning days, the rats were placed into the operant boxes, and after response rates stabilized, a 10-min baseline recording period commenced. At the end of this baseline period, and 3 min prior to drug or water injection, the audiovisual CS was presented. This CS consisted of (1) offset of the background masking noise and the onset of a $5-\mathrm{Hz}, 65-\mathrm{dB}$ clicking sound and (2) turning off the operant chamber "houselight" and the illumination of two red and orange "cue lamps" located directly above the response lever.

During the 3-min CS period, an observer who was "blind" to the group assignment of each subject conducted a time-sampling visual monitoring of the rats by means of the small aperture and wide angle lens mounted in each Skinner box enclosure. Each of the 3 rats was observed for three 15 -sec periods (with about 40 sec elapsing between subsequent sampling periods for each subject), so that each subject was observed for a total of $45 \mathrm{sec}$. The observer counted the number of times the rat reared at (stood on hind legs and extended forepaws against) any of the three walls nearest the response lever, and also the number of excursions the rat made across a center line bisecting the longer lateral dimension $(30.5 \mathrm{~cm})$ of the operant chamber. The rearing and crossing frequencies were accumulated for all three 15-sec periods for each rat for each CS presentation, the intent being to quantify the observed frequency of lo- 
comotion away from the immediate vicinity of the response lever. No attempt was made to measure the duration of such activity.

Three minutes after CS onset, response-rate recording ceased while each rat was briefly removed from the operant box and administered an i.p. injection of $15 \mathrm{mg} / \mathrm{kg}$ sodium pentobarbital (experimental group) or a water injection of equivalent volume (control group) and immediately replaced in the operant box. Three rats were run simultaneously, and the entire injection procedure for all 3 required no more than $90 \mathrm{sec}$. The CS continued and response rate was recorded for the remaining $20 \mathrm{~min}$ of the operant session.

Two hours after the end of the session, the control group animals were given i.p. injections of $15 \mathrm{mg} / \mathrm{kg}$ sodium pentobarbital in their home cages, and the experimental group rats were given water injections. This delayed injection of the controls ensured that any differences between the two groups were not due to chronic pentobarbital treatment effects not involving association between drug treatment and the CS. The $15-\mathrm{mg} / \mathrm{kg}$ dose was chosen after pilot work indicated that this was the minimal dose that reliably stopped e'sentially all responding prior to the end of the 20 -min postinjection period.

Each conditioning day was followed by a nonconditioning day on which the rats worked for food pellets for $20 \mathrm{~min}$ on the VI $60-\mathrm{sec}$ reinforcement schedule, but no CS was presented and no injections were given. These nonconditioning sessions prevented the overall test environment from becoming conditioned to the drug effects in the experimental animals.

Test for CR to injection procedure. On the day following the 12 th conditioning session, a final session was conducted that was identical to the sessions on the previous conditioning days except for the following procedural changes: Both groups were administered water injections, and 3-min visual observations (the same as in the preinjection period on previous conditioning days) were made starting 2,9 , and 16 min after the injection.

\section{RESULTS}

Mean baseline response rates for both groups over the 12 conditioning days were within the range of 11 to 15 responses per minute. All results involving response rates are expressed as percentage of the 10-min pre-CS baselines. The barbiturate injection produced almost complete cessation of responding within $5 \mathrm{~min}$. The postinjection visual observations revealed that response suppression was due to severe ataxia, followed by sedation and markedly reduced activity that persisted throughout the 20min postinjection period. The water injection produced a slight decrease from the baseline rate in the control subjects. The dramatic difference between the experimental and control groups persisted without detectible systematic change over the course of the 12 conditioning days. The nearly complete suppression of responding produced by the drug injection in the experimental group did not abate during the course of conditioning. Group means of percent of baseline response rates for the entire 20-min postinjection period for 3 representative conditioning days $(\mathrm{CD})$ were as follows for the experimental $(\mathrm{EX})$ and control (C) rats: $\mathrm{CD} 1 \mathrm{EX}=12 \%, \mathrm{C}=73 \%$; $\mathrm{CD} 6 \mathrm{EX}=$ $14 \%, \mathrm{C}=81 \% ; \mathrm{CD} 12 \mathrm{EX}=9 \%, \mathrm{C}=84 \%$.

Figure 1 illustrates the development of the conditioned suppression of responding in response to the preinjection audiovisual CS. A 2 (treatment groups) $\times 13$ (12 conditioning days +1 extinction/test day) analysis of variance (ANOVA) revealed a significant main group effect $[F(1,15)$ $=6.26, p<.05]$ and days effect $[F(12,180)=2.51, p<$

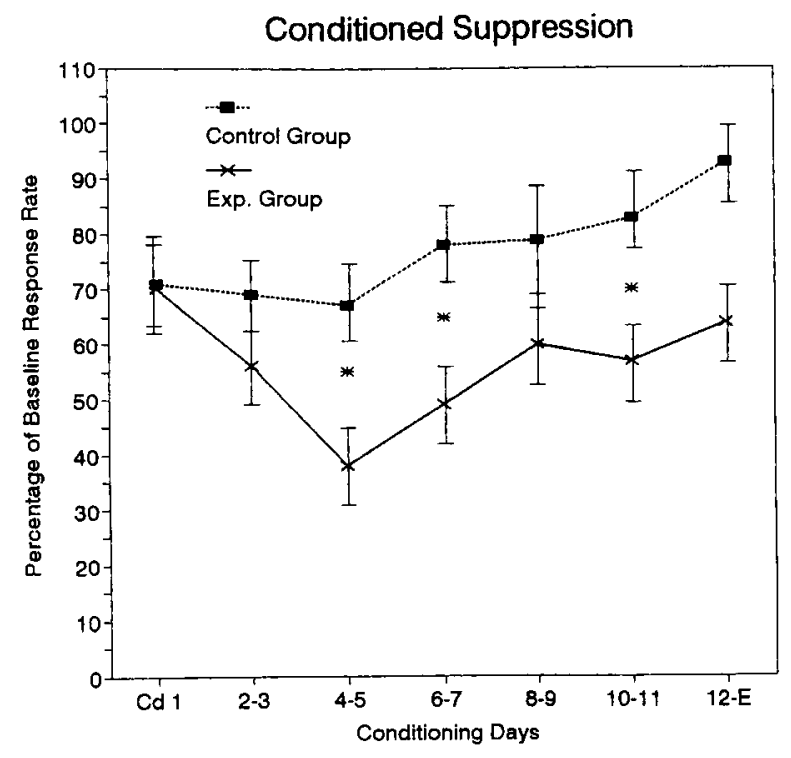

Figure 1. Conditioned response as indicated by suppression of operant responding (mean percent change from baseline response rate) to 3-min audiovisual stimuli preceding pentobarbital or water injection over 12 conditioning days plus the extinction day. *Significant differences.

$.01]$, but no significant interaction. The control group animals exhibited a slight suppression during early conditioning sessions, which became less apparent during later sessions. The experimental group animals displayed increasing conditioned suppression to the preinjection $\mathrm{CS}$, which was greatest on CDs 4 and 5. There was some tendency toward less suppression during later sessions, in that significant group differences were seen on $\mathrm{CDs} 4-5$, 6-7, and 10-11, but not on CDs 8-9 and 12-13 (Day 13 was actually the test day, but up to the point of injection, procedures were identical to those on previous conditioning days). The significance of these differences was determined by Tukey's tests.

Figure 2 illustrates the mean frequencies of locomotor activity incompatible with leverpressing that occurred during the preinjection CS periods, as determined by the time-sampling visual observations. A $2 \times 13$ ANOVA indicated a significant main group effect $[F(1,15)=$ $9.12, p<.01]$ and a significant group $\times$ days interaction $[F(12,180)=1.91, p<.05]$, but no significant main days effect. The pattern of group differences seen in the visualobservation data was generally similar to that of the operant suppression data. During the initial conditioning (CDs 1-3), the experimental and control groups' locomotor activity leading away from the response lever was not significantly different. Tukey's tests showed that significant differences did emerge by CDs 4-5, and the significantly greater amount of this activity in the experimental group persisted throughout all remaining CDs.

Visual observation of the rats during the preinjection CS periods gave no indication that the moderate degree of conditioned suppression occurring during these periods was due to conditioned ataxia or other indicators of 


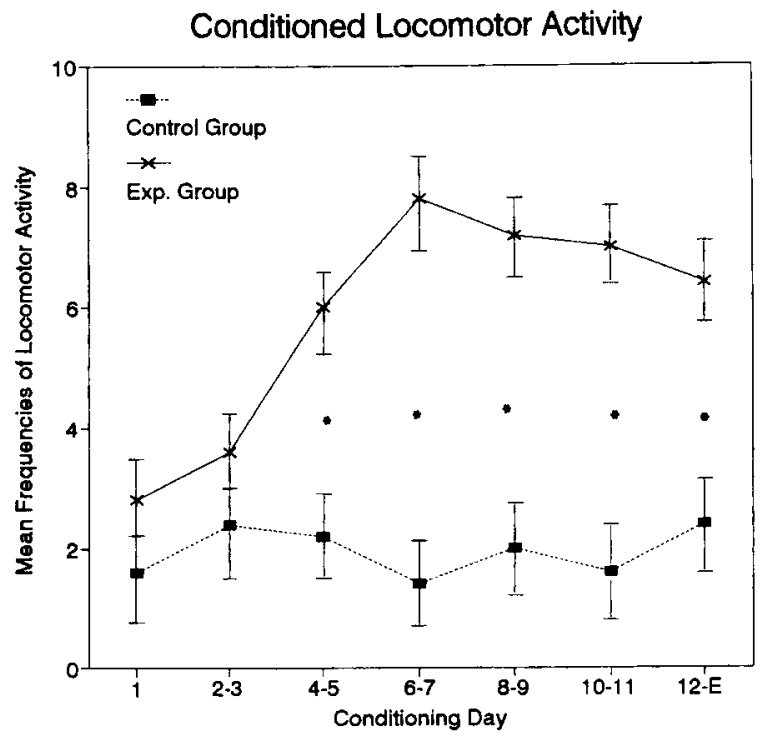

Figure 2. Conditioned response as indicated by locomotor activity incompatible with leverpressing (mean frequencies of locomotor activity) elicited by 3 -min audiovisual stimuli preceding pentobarbital or water injection over 12 conditioning days plus the extinction day. *Significant differences.

behavioral depression. These observations did suggest that the animals were somewhat aroused, in that the CS elicited locomotor activity, rearing, and other behaviors that were incompatible with and thus lowered the rate of leverpressing below baseline levels.

Figure 3 demonstrates the absence of a detectable conditioned change in response rate resulting from the injection procedure. The control and experimental groups' postinjection responses were very similar after both received water injections on the extinction/test day. Visual monitoring of locomotor activity for three 3 -min postinjection periods on the test day also failed to provide convincing evidence of a postinjection CR. Mean values for occurrence of these behaviors during the first observation period were $4.9( \pm .9)$ for the control group and 5.6 $( \pm 1.4)$ for the experimental group. Group differences for the remaining two observation periods were even smaller, and an ANOVA of these data revealed no significant main or interaction effects.

\section{DISCUSSION}

The results of this experiment show that in the operant paradigm, under the conditions described, the $\mathrm{CR}$ elicited by a CS predicting the onset of barbiturate effects results in a decrease in operant response rate from pre-CS baseline levels. Since the unconditioned response to the barbiturate injection was also a marked reduction in response rate, the CR was "isodirectional" in relation to the UR, and these results are thus to some extent consistent with previous research in which conditioned drug effects have been studied in the operant paradigm. However, the sup- plementary visual observation of the behaviors elicited by the preinjection $\mathrm{CS}$ revealed that these responses were quite different from the ataxia and sedation produced by the drug injection. The decrease in response rate during the preinjection CS period was concurrent with increased frequency of locomotor activity, and the rats' movement away from the response lever. Therefore, although the effect on response rate was similar (in the same direction) for both the conditioned and unconditioned responses, it seems more appropriate to describe the basic CR as "drugoppositional," rather than "drug-like."

In the previous experiment (Watanabe, 1990) in which both conditioned and unconditioned barbiturate effects in the operant paradigm caused increased response rate, the drug dose was lower and the reinforcement schedule was FI. The influence of conditioned responses on operant responding, like essentially all drug effects, is no doubt dose and reinforcement-schedule specific.

Drug-oppositional conditioned effects, causing increased levels of activity, have been demonstrated with morphine as the US (Hinson \& Siegel, 1983; Mucha, Volkovsiks, \& Kalant, 1981; Paletta \& Wagner, 1986). The results presented here may be the first to demonstrate such a conditioned effect with barbiturate treatment. Conditioned compensatory responses have been demonstrated with another CNS depressant (ethanol) and a different response (body temperature changes; Cunningham \& Schwarz, 1989; Le, Poulos, \& Cappell, 1979; Mansfield \& Cunningham, 1980).

This experiment is unique in the use of a preinjection $\mathrm{CS}$, which enabled daily observation of the CRs as they developed, rather than only on an extinction-test day. The preinjection $\mathrm{CR}$, as indicated by operant response suppression, was fully developed by CDs $4-5$ and appeared to attenuate to some extent during subsequent CDs. Group differences in degree of suppression were not significant for CD Blocks 8-9 and 12-13.

The locomotor-activity data reflect a pattern parallel (but inverse) to that of the operant suppression results. However, the attenuation of the locomotor CR during

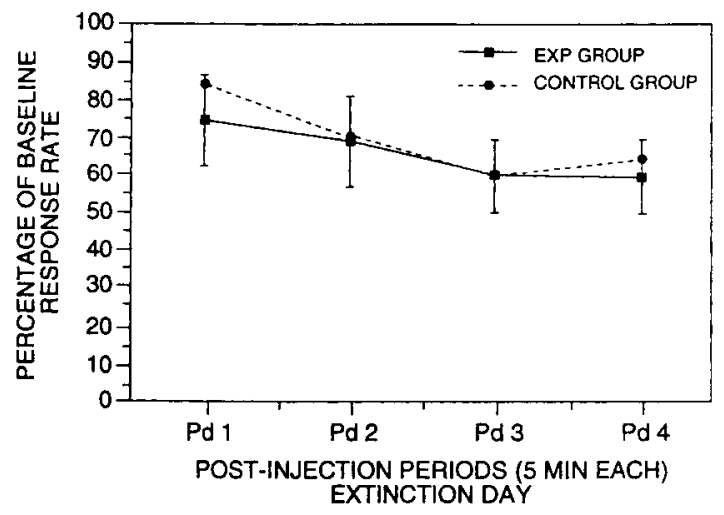

Figure 3. Postinjection response (mean percent change from baseline response rate) to water injection on extinction day. 
later conditioning sessions was less apparent in that significant group differences were seen consistently after CD Block 4-5. This tendency for a decrease in strength of both conditioned effects as the treatment days progressed may have arisen from the development of tolerance to the behavioral disruptive effect of the increased locomotor activity on the ongoing operant behavior. Behavioral tolerance seems to be a result of both instrumental learning (Wolgin, 1989) and Pavlovian conditioning (Cunningham, Losli, \& Risinger, 1992), and either or both processes could have been involved here.

One difference between the present experiment and the similar previous experiment with amphetamine (Duncan et al., 1989) is that there was no tendency for attenuation of the preinjection conditioned operant suppression in the later conditioning trials at amphetamine doses of 1.5 and $3 \mathrm{mg} / \mathrm{kg}$, although such a tendency was seen at the $.8-\mathrm{mg} / \mathrm{kg}$ dose.

There was no evidence for conditioning to the injection and handling procedure as assessed by suppression of response rate and visual observation during the postinjection period on the test day. The absence of conditioned effects in response to these cues is somewhat surprising given the occurrence of preinjection conditioned effects. However, the preinjection CS may have reduced the information value (i.e., the "predictiveness") of the injection procedure, which occurred $3 \mathrm{~min}$ after the onset of the audiovisual CS component. In the terminology of Kamin (1969), the first stimulus may have been more salient and thus "overshadowed" the second stimulus. Coupled with the overshadowing aspect of the sequence of CS presentation is the partial reinforcement effect (Mackintosh, 1974). For every barbiturate injection there was also a water injection (administered $2 \mathrm{~h}$ later in the home cage for the experimental group), so half of the injections were not immediately followed by the onset of drug effects. Essentially, the injection procedure was neither a reliable nor a unique predictor of barbiturate action.

The UR elicited by the injection of $15 \mathrm{mg} / \mathrm{kg}$ of sodium pentobarbital did not become noticeably weaker across the 12 days of conditioning. This result is somewhat surprising in that an opposite-direction CR (to the preinjection CS) was seen, and such "compensatory" responses are thought to contribute to the overall development of tolerance. Perhaps different experimental conditions (dose, time between drug treatments, etc.) or a different method of measuring the drug effects would have revealed the development of tolerance, which was not seen in either the operant behavior or in the observed locomotor behavior.

Finally, Siegel (1989) has pointed out that environmentspecific drug tolerance, supposedly resulting from classical conditioning of compensatory responses, is a very reliable phenomenon, seen with several CNS depressant and opiate drugs under widely varying experimental conditions (see, e.g., Cappell, Roach, \& Poulos, 1981; Hinson \& Siegel, 1986; Melchior \& Tabakoff, 1985; Vila, 1989). However, the observation of such compensatory responses per se, in situations not involving tolerance to the drug, is less reliable, and in general is a less robust effect, with several researchers reporting no evidence of such responses. The results presented here indicate that the operant paradigm is one in which conditioned "compensatory" responses can be demonstrated, in particular when responses in addition to rate of the operant response are considered in describing the overall nature of the elicited behavior.

\section{REFERENCES}

Cameron, O., \& APPEL, J. B. (1972). Conditioned suppression of barpressing behavior by stimuli associated with drugs. Journal of the Experimental Analysis of Behavior, 17, 127-137.

Cappell, H., Roach, C., \& Poulos, C. X. (1981). Pavlovian control of cross-tolerance between pentobarbital and ethanol. Psychopharmacology, 74, 54-57.

Cunningham, C., Losli, S., \& Risinger, F. (1992). Context-drug pairings enhance tolerance to ethanol-induced disruption of operant responding. Psychopharmacology, 109, 217-222.

Cunningham, C., \& Schwarz, K. (1989). Pavlovian-conditioned changes in body temperature induced by alcohol and morphine. Drug Development Research, 16, 295-303.

Duncan, P., Barry, T., Ellis, R., \& Hinkle, E. (1989). Conditioned response to amphetamine injection with the operant paradigm. Drug Development Research, 16, 133-141.

Duncan, P., \& Koontz, K. (1994). Pavlovian conditioning of insulinproduced motor activity and blood-glucose responses. Behavioural Pharmacology, 5(Suppl. 1), 65.

EIKELBOOM, R., \& STEWART, J. (1982). Conditioning of drug-induced physiological responses. Psychological Review, 89, 507-528.

ESTES, K., \& SKINNER, B. (1941). Some quantitative properties of anxiety. Journal of Experimental Psychology, 29, 390-397.

GoldberG, S., \& Schuster, C. (1967). Conditioned suppression by a stimulus associated with nalorphine in morphine-addicted monkeys. Journal of the Experimental Analysis of Behavior, 10, 235-242.

Hinson, R. E., \& SiEgel, S. (1983). Anticipatory hyperexcitability and tolerance to the narcotizing effect of morphine in the rat. Behavioral Neuroscience, 97, 759-767.

Hinson, R. E., \& SiEgel, S. (1986). Pavlovian inhibitory conditioning and tolerance to pentobarbital-induced hypothermia in rats. Journal of Experimental Psychology: Animal Behavior Processes, 12, 363 370.

KAMIN, L. J. (1969). Predictability, surprise, attention, and conditioning. In B. Campbell \& R. Church (Eds.), Punishment and aversive behavior (pp. 279-296). New York: Appleton-Century-Crofts.

Le, A. D., Poulos, C. X., \& CAPPEll, H. (1979). Conditioned tolerance to the hypothermia effect of ethyl alcohol. Science, 206, 1109-1110.

Lubow, R. E. (1973). Latent inhibition. Psychological Bulletin, 79, 398-407.

Mackintosh, N. J. (1974). The psychology of animal learning. London: Academic Press.

Mansfield, J. G., \& Cunningham, C. L. (1980). Conditioning and extinction of tolerance to the hypothermic effect of ethanol in rats. Journal of Comparative \& Physiological Psychology, 94, 962-969.

MelChior, C., \& TABAKOFF, B. (1985). Features of environmentdependent tolerance to ethanol. Psychopharmacology, 87, 94-100.

Mucha, R. F., Volkovsiks, C., \& Kalant, H. (1981). Conditioned increases in locomotor activity produced with morphine as an unconditioned stimulus, and the relation of conditioning to acute morphine effect and tolerance. Journal of Comparative \& Physiological Psychology, 95, 351-362.

Paletta, M.S., \& Wagner, A. R. (1986). Development of contextspecific tolerance to morphine: Support for a dual-process interpretation. Behavioral Neuroscience, 100, 611-623.

SIEGEL, S. (1975). Conditioning insulin effects. Journal of Comparative \& Physiological Psychology, 89, 498-506.

SiEgel, S. (1983). Classical conditioning, drug tolerance, and drug dependence. In Y. Israel, F. Glaser, H. Kalant, R. Popham, W. Schmidt, 
\& R. Smart (Eds.), Research advances in alcohol and drug problems (Vol. 7, pp. 207-246). New York: Plenum.

SIEGEL, S. (1989). Pharmacological conditioning and drug effects. In A. J. Goudie \& M. W. Emmett-Ogelsby (Eds.), Psychoactive drugs. Tolerance and sensitization (pp. 115-180). Clifton, NJ: Humana Press.

Stewart, J., \& Eikelboom, R. (1987). Conditioned drug effects. In L. Iversen, S. Iversen, \& S. Snyder (Eds.), Handbook of psychopharmacology (Vol. 19, pp. 65-143). New York: Plenum.

VILA, C. (1989). Protection from pentobarbital lethality mediated by Pavlovian conditioning. Pharmacology, Biochemistry \& Behavior, 32, 365-366.

WATANABE, S. (1990). Isodirectional conditioning effects of $d$-amphetamine and pentobarbital on schedule-controlled operant behavior in pigeons. Pharmacology, Biochemistry \& Behavior, 36, 157-161.
WhitNey, G., \& Trost, J. (1970). Response disruption following amphetamine self- and programmed administration. In R. Harris, W. McIsaac, \& C. Schuster (Eds.), Drug dependence (pp. 198-213). Austin: University of Texas Press.

Wolgin, D. L. (1989). The role of instrumental learning in behavioral tolerance to drugs. In A. J. Goudie \& M. W. Emmett-Ogelsby (Eds.), Psychoactive drugs: Tolerance and sensitization (pp. 17-114). Clifton, NJ: Humana Press.

(Manuscript received February 6, 1996; revision accepted for publication November 29,1996 .) 